\title{
Proca equations derived from first principles
}

\author{
Michel Gondran \\ Université Paris Dauphine, 75016 Paris, France.*
}

\begin{abstract}
Gersten has shown how Maxwell equations can be derived from first principles, similar to those which have been used to obtain the Dirac relativistic electron equation. We show how Proca equations can be also deduced from first principles, similar to those which have been used to find Dirac and Maxwell equations.

Contrary to Maxwell equations, it is necessary to introduce a potential in order to transform a second order differential equation, as the Klein-Gordon equation, into a first order differential equation, like Proca equations.
\end{abstract}




\section{INTRODUCTION}

The Schrödinger and Klein-Gordon equations can be directly deduced from first principles, i.e. from the condition liking the Energy $E$, mass $m$, and momentum $\overrightarrow{\mathbf{p}}$ and from the correspondence principle where $E$ and $\overrightarrow{\mathbf{p}}$ are substituted by the quantum operators $i \hbar \frac{\partial}{\partial t}$ and $-i \hbar \nabla$. The non relativistic condition $\left(E-\frac{\overrightarrow{\mathbf{p}}^{2}}{2 m}-V\right) \psi=0$ where $\psi$ is a wavefunction gives the Schrödinger equation and the relativistic condition $\left(E^{2}-c^{2} \overrightarrow{\mathbf{p}}^{2}-m^{2} c^{4}\right) \psi=0$ gives the Klein-Gordon equation.

The Dirac relativistic electron equation ${ }^{\underline{1}}$ is also derived from first principles, but indirectly with a four component wavefunction which permits to introduce a spin- $\frac{1}{2}$ particle.

Gersten has shown 2 how Maxwell equations can be obtained from first principles, similar to those which have been used to infere the Dirac equation ${ }^{1}$.

The aim of the present paper is to show how Proca equations $3 \frac{3.4}{4}$, describing a massive spin-1 particle, can be also derived from first principles, using a decomposition similar to those which was used to find Dirac and Maxwell equations. We can notice that the Proca equations can be used for massive photons $\frac{4,5}{5}$ and for the London penetration depth in a superconductor $\stackrel{4.6}{\underline{n}}$.

Contrary to Maxwell equations, it is necessary to introduce a potential in order to transform a second order differential equation, as the Klein-Gordon equation, into a first order differential equation, as the Proca equations.

The method of Dirac and Gerster to obtained the Dirac and Maxwell equations are recall in Section 2. The method to obtain the Proca equations is described in Section 3.

\section{DIRAC AND MAXWELL EQUATIONS}

In his 1928 seminal paper ${ }^{1}$, Dirac deduces his equation from the relativistic condition liking the Energy $E$, mass $m$, and momentum $\overrightarrow{\mathbf{p}}$ :

$$
\left(E^{2}-c^{2} \overrightarrow{\mathbf{p}}^{2}-m^{2} c^{4}\right) I^{(4)} \Psi=0
$$

where $I^{(4)}$ is the $4 \times 4$ unit matrix and $\Psi$ is a four component column (bispinor) wavefunction. 
Dirac decomposes Eq. (11) into

$$
\left[E I^{(4)}+\left(\begin{array}{cc}
m c^{2} I^{(2)} & c \overrightarrow{\mathbf{p}} \cdot \vec{\sigma} \\
c \overrightarrow{\mathbf{p}} \cdot \vec{\sigma} & -m c^{2} I^{(2)}
\end{array}\right)\right]\left[E I^{(4)}-\left(\begin{array}{cc}
m c^{2} I^{(2)} & c \overrightarrow{\mathbf{p}} \cdot \vec{\sigma} \\
c \overrightarrow{\mathbf{p}} \cdot \vec{\sigma} & -m c^{2} I^{(2)}
\end{array}\right)\right] \Psi=0
$$

where $I^{(2)}$ is the $2 \times 2$ unit matrix and $\vec{\sigma}$ is the Pauli spin one-half vector matrix with the components $\sigma_{x}=\left(\begin{array}{ll}0 & 1 \\ 1 & 0\end{array}\right), \sigma_{y}=\left(\begin{array}{cc}0 & -i \\ i & 0\end{array}\right)$ et $\sigma_{z}=\left(\begin{array}{cc}1 & 0 \\ 0 & -1\end{array}\right)$.

Eq. (2) will be satisfied if the equation

$$
\left[E I^{(4)}-\left(\begin{array}{cc}
m c^{2} I^{(2)} & c \overrightarrow{\mathbf{p}} \cdot \vec{\sigma} \\
c \overrightarrow{\mathbf{p}} \cdot \vec{\sigma} & -m c^{2} I^{(2)}
\end{array}\right)\right] \Psi=0
$$

will be satisfied. The Dirac equation will be obtained by substitution in the equation (3) of $E$ and $\overrightarrow{\mathbf{p}}$ by the quantum operators $i \hbar \frac{\partial}{\partial t}$ and $-i \hbar \nabla$.

The two component neutrino equation can be infered, in the same manner, from the decomposition

$$
\left[E^{2}-c^{2} \overrightarrow{\mathbf{p}}^{2}\right) I^{(2)} \Psi=\left(E I^{(2)}-c \overrightarrow{\mathbf{p}} \cdot \vec{\sigma}\right]\left[E I^{(2)}+c \overrightarrow{\mathbf{p}} \cdot \vec{\sigma}\right] \Psi=0
$$

where $\Psi$ is a two component spinor wavefunction.

Gersten $^{2}$ finds in 1998 the photon equation from the relativistic condition on the Energy $E$ and momentum $\overrightarrow{\mathbf{p}}$ :

$$
\left(\frac{E^{2}}{c^{2}}-\overrightarrow{\mathbf{p}}^{2}\right) I^{(3)} \vec{\Psi}=0,
$$

where $I^{(3)}$ is the $3 \times 3$ unit matrix and $\vec{\Psi}$ is a three component column wavefunction.

Gersten decomposes Eq. (5) into

$$
\left[\frac{E}{c} I^{(3)}-\overrightarrow{\mathbf{p}} \cdot \overrightarrow{\mathbf{S}}\right]\left[\frac{E}{c} I^{(3)}+\overrightarrow{\mathbf{p}} \cdot \overrightarrow{\mathbf{S}}\right] \vec{\Psi}-\left(\begin{array}{c}
p_{x} \\
p_{y} \\
p_{z}
\end{array}\right)(\overrightarrow{\mathbf{p}} \cdot \vec{\Psi})=0
$$

where $\overrightarrow{\mathbf{S}}$ is a spin one vector matrix with components

$$
S_{x}=\left(\begin{array}{ccc}
0 & 0 & 0 \\
0 & 0 & -i \\
0 & i & 0
\end{array}\right), \quad S_{y}=\left(\begin{array}{ccc}
0 & 0 & i \\
0 & 0 & 0 \\
-i & 0 & 0
\end{array}\right), \quad S_{z}=\left(\begin{array}{ccc}
0 & -i & 0 \\
i & 0 & 0 \\
0 & 0 & 0
\end{array}\right),
$$


and with the properties

$$
\left[S_{x}, S_{y}\right]=i S_{z}, \quad\left[S_{z}, S_{x}\right]=i S_{y}, \quad\left[S_{y}, S_{z}\right]=i S_{x}, \quad \overrightarrow{\mathrm{S}}^{2}=2 I^{(3)} .
$$

Eq. (66) will be satisfied if the two equations

$$
\begin{gathered}
{\left[\frac{E}{c} I^{(3)}+\overrightarrow{\mathbf{p}} \cdot \overrightarrow{\mathrm{S}}\right] \vec{\Psi}=0,} \\
\overrightarrow{\mathbf{p}} \cdot \vec{\Psi}=0,
\end{gathered}
$$

will be simultaneously satisfied. The Maxwell equations will be obtained by substitution in Eqs. (9, 10) of $E$ and $\overrightarrow{\mathrm{p}}$ by the quantum operators $i \hbar \frac{\partial}{\partial t}$ and $-i \hbar \nabla$, and the wavefunction substitution

$$
\vec{\Psi}=\overrightarrow{\mathbf{E}}-i \overrightarrow{\mathbf{B}}
$$

where $\overrightarrow{\mathbf{E}}$ and $\overrightarrow{\mathbf{B}}$ are the electric and magnetic fields respectively. With the identity

$$
(\overrightarrow{\mathbf{p}} \cdot \overrightarrow{\mathrm{S}}) \vec{\Psi}=\hbar \nabla \times \vec{\Psi}
$$

Eqs. (9) and (10) give

$$
\begin{gathered}
i \frac{\hbar}{c} \frac{\partial(\overrightarrow{\mathbf{E}}-i \overrightarrow{\mathbf{B}})}{\partial t}=-\hbar \nabla \times(\overrightarrow{\mathbf{E}}-i \overrightarrow{\mathbf{B}}), \\
\nabla \cdot(\overrightarrow{\mathbf{E}}-i \overrightarrow{\mathbf{B}})=0 .
\end{gathered}
$$

There are exactly all the Maxwell equations if the electric and magnetic fields are real.

\section{PROCA EQUATIONS}

In this 1936 paper $^{3}$, Proca gives relativistic wave equations for a massive spin-1 particle. We derive these equations from the relativistic condition on the Energy $E$, mass $m$, and momentum $\overrightarrow{\mathbf{p}}$ :

$$
\left(\frac{E^{2}}{c^{2}}-\overrightarrow{\mathbf{p}}^{2}-m^{2} c^{2}\right) I^{(3)} \vec{\Psi}=0,
$$

where $I^{(3)}$ is the $3 \times 3$ unit matrix and $\vec{\Psi}$ is a three component column wavefunction.

Eq. (15) can be decomposed into

$$
\left[\frac{E}{c} I^{(3)}-\overrightarrow{\mathbf{p}} \cdot \overrightarrow{\mathbf{S}}\right]\left[\frac{E}{c} I^{(3)}+\overrightarrow{\mathbf{p}} \cdot \overrightarrow{\mathbf{S}}\right] \vec{\Psi}-\left(\begin{array}{c}
p_{x} \\
p_{y} \\
p_{z}
\end{array}\right)(\overrightarrow{\mathbf{p}} \cdot \vec{\Psi})-m^{2} c^{2} \vec{\Psi}=0
$$


where $\overrightarrow{\mathrm{S}}$ is a spin one vector matrix defined by Eqs (7) and (8).

Then we define the potential $(\varphi, \overrightarrow{\mathbf{A}})$ by the following equations:

$$
\begin{gathered}
\overrightarrow{\mathbf{p}} \cdot \vec{\Psi}=i \frac{m^{2} c^{2}}{\hbar} \varphi \\
{\left[\frac{E}{c} I^{(3)}+\overrightarrow{\mathbf{p}} \cdot \overrightarrow{\mathbf{S}}\right] \vec{\Psi}=i \frac{m^{2} c^{2}}{\hbar} \overrightarrow{\mathbf{A}}}
\end{gathered}
$$

If we use these two values in the Eq.(16) and substitute $E$ and $\overrightarrow{\mathbf{p}}$ by the quantum operators $i \hbar \frac{\partial}{\partial t}$ and $-i \hbar \nabla$, we find the equation which decribe the wavefunction from the potential

$$
\vec{\Psi}=-\frac{1}{c} \frac{\overrightarrow{\mathrm{A}}}{\partial t}-i \nabla \times \overrightarrow{\mathbf{A}}-\nabla \cdot \varphi
$$

The other equations correspond to Eqs.(17) and (18) by substitution of $E$ and $\overrightarrow{\mathbf{p}}$ by the quantum operators $i \hbar \frac{\partial}{\partial t}$ and $-i \hbar \nabla$

$$
\begin{gathered}
\nabla \vec{\Psi}=-\frac{m^{2} c^{2}}{\hbar^{2}} \varphi, \\
\frac{1}{c} \frac{\partial \vec{\Psi}}{\partial t}-i \nabla \times \vec{\Psi}=\frac{m^{2} c^{2}}{\hbar^{2}} \overrightarrow{\mathbf{A}} .
\end{gathered}
$$

If we put $\vec{\Psi}=\overrightarrow{\mathbf{E}}-i \overrightarrow{\mathbf{B}}$, we deduce from Eqs.(19),(20) and (21), if $\overrightarrow{\mathbf{E}}$ and $\overrightarrow{\mathbf{B}}$ are real, the Proca equations

$$
\begin{gathered}
\overrightarrow{\mathbf{E}}=-\frac{1}{c} \frac{\partial \overrightarrow{\mathbf{A}}}{\partial t}-\nabla \cdot \varphi, \quad \overrightarrow{\mathbf{B}}=\nabla \times \overrightarrow{\mathbf{A}} \\
\nabla \cdot \overrightarrow{\mathbf{E}}=--\frac{m^{2} c^{2}}{\hbar^{2}} \varphi, \quad \nabla \cdot \overrightarrow{\mathbf{B}}=0 \\
\frac{1}{c} \frac{\partial \overrightarrow{\mathbf{E}}}{\partial t}-\nabla \times \overrightarrow{\mathbf{B}}=\frac{m^{2} c^{2}}{\hbar^{2}} \overrightarrow{\mathbf{A}}, \quad \frac{1}{c} \frac{\partial \overrightarrow{\mathbf{B}}}{\partial t}+\nabla \times \overrightarrow{\mathbf{E}}=0
\end{gathered}
$$

\section{CONCLUSION}

Above, we have shown how all Proca equations, Eqs. (22), (23) and (24), can be obtained from first principles, similar to those which have been used to find Dirac and Maxwell equations.

As demonstrated by Lévy-Leblond ${ }^{7}$, the spin of the Dirac electron is not of relativistic origin, but primarily a consequence of the use of a multicomponent wavefunction. It is the same for the Maxwell and Proca particles. 
The Dirac, Maxwell and Proca wavefunction are complex. Moreover the Maxwell and Proca wave functions $\vec{\Psi}=\overrightarrow{\mathbf{E}}-i \overrightarrow{\mathbf{B}}$ are locally measurable and well understood quantities. Therefore these wavefunctions should be used as a guideline for proper interpretations of quantum theories.

* Electronic address: michel.gondran@polytechnique.com

1 P. Dirac: "The Quantum Theory of the Electron", Proc. Roy. Soc. A117, 620 (1928). (1928).

2 A. Gersten: "Maxwell equations as the one-photon quantum equation". Found. Phys. Lett. 12, 291-8 (1998) .

3 A. Proca, Le Journal de Physique et le Radium 7, 347 (1936).

4 J. D. Jackson, Classical Electrodynamics, John Wiley and Sons, New York, 1999.

5 T. Prokopec and R. Woodard, "Vacuum polarization and photon mass in inflation", Am. J. Phys. $72,60-72(2004)$.

6 L. H. Ryder, Quantum Field Theory, Cambridge University Press, Cambridge, 2003.

7 J. M. Lévy-Leblond, Comm. Math. Phys. 6, 286 (1967). 